\title{
Focusing surtace waves using an axicon
}

\author{
Hayrettin Köymen and Abdullah Atalar \\ Middle East Technical University, Electrical and Electronic Engineering Department, Ankara, Turkey
}

(Received 29 July 1985; accepted for publication 14 October 1985)

\begin{abstract}
Axicons are generators of waves which focus on a line. They are used in various imaging and nondestructive testing applications as bulk wave focusing devices with a very long depth of focus. In this letter, a new type of conical axicon is introduced and it is shown that this axicon, immersed in a liquid, insonifying a plane solid surface can be used to excite surface waves on the solid surface provided that the cone angle of the axicon coincides with the Rayleigh critical angle of the liquidsolid interface. The generated surface waves focus into a diffraction-limited spot. This new surface wave focusing scheme is easy to use, has a conversion efficiency and sensitivity far better than other existing techniques.
\end{abstract}

The problem of generating surface acoustic waves (SAW's) in nonpiezoelectric materials has been extensively studied and various techniques exist. ${ }^{1.2}$ Use of wedge transducers is one of the most prominent. Oblique incidence of a bulk wave at an appropriate angle generates surface waves on the surface of interest. Using this technique one obtains high conversion efficiency ${ }^{3}$ from the bulk wave into a SAW. The generated SAW is collimated with a width determined by the width of the wedge. Such a configuration is suitable for detection of surface irregularities such as cracks. On the other hand, to characterize or image the defect rather than merely detecting it requires a more laterally confined or a focused surface wave. Such a wave will not only give better resolution but also will result in higher sensitivity. Tuan $e t$ $a l .{ }^{4}$ used a phased array edge coupled transducers to achieve this task.

Smith et al..$^{5}$ have proposed a method of generating convergent SAW's on the surface to be examined by defocusing a spherical acoustic microscope lens employing a semicircular disk transducer. The generated SAW focuses on a spot coinciding with axis of the lens. They have shown that an acoustic image taken by a regular acoustic microscope in defocused condition is indeed mostly a focused SAW image. The principal drawback in their system is that the amount of energy converted into SAW's is limited to the energy emitted from a very narrow strip on the surface of the lens.

Another method is proposed by Nongaillard et al. to overcome the disadvantages of this system. They use a cylindrical lens tilted at the Rayleigh critical angle to excite focused SAW's. ${ }^{6}$ A greater bulk to SAW conversion efficiency is obtained in this system, but still not all of the incident rays are at Rayleigh critical angle and the SAW energy is spread over a line rather than focused on a spot.

In this letter, we introduce a novel method of focusing SAW's on the surface of nonpiezoelectric materials by using a new type of conical axicon. The axicon, which is the name commonly given to generators of waves which focus on a line, has been proposed as a bulk acoustic wave focusing device. ${ }^{7}$ They have extended depth of focus, but suffers from inherent off-axis sensitivity and the difficulty of manufacturing.

Consider the geometry shown in Fig. 1. An acoustic beam generated by a piston transducer in the liquid medium is obliquely incident on a concave parabolic cylinder surface of a solid. If the incidence angle is high enough, the surface will act like a parabolic cylinder mirror.

Recalling that a parabolic cylinder mirror focuses a normally incident plane wave into a line focus, an obliquely incident plane wave will be focused into a line with a linearly varying phase. This type of line focus was obtained earlier using conical axicons; thus the arrangement shown in Fig. 1 is a new type of conical axicon. The reflected wave fronts are conical and in our case the axis of the cone coincides with the focal line of the parabolic cylinder.

Now suppose that a material with a plane surface is placed perpendicular to this axis. The intersection of the conical wave fronts with the material surface is always circular. Note that the arrangement proposed by Nongaillard $e t$ $a l .{ }^{6}$ involves oblique cylindrical wave fronts and in this case, the intersection with the material surface would be elliptical instead of circular, hence it will not converge to a spot.

It is well known that ${ }^{8}$ a beam incident on a liquid-solid interface excites surface waves strongly provided that the incidence angle is equal to the Rayleigh critical angle. We can combine this fact with our ultrasonic axicon to generate a focused surface wave: the incidence angle of the beam created by the transducer is adjusted to equal the Rayleigh critical angle. In this case, all the reflected rays from the mirror will hit the solid surface at the Rayleigh critical angle. This fact is easily proven considering the direction cosines. ${ }^{9}$ As the conical wave front propagates towards the interface, the intersection with the material surface will be a circular arc with diminishing radius. The excited surface wave will reinforce the surface wave front previously excited when the

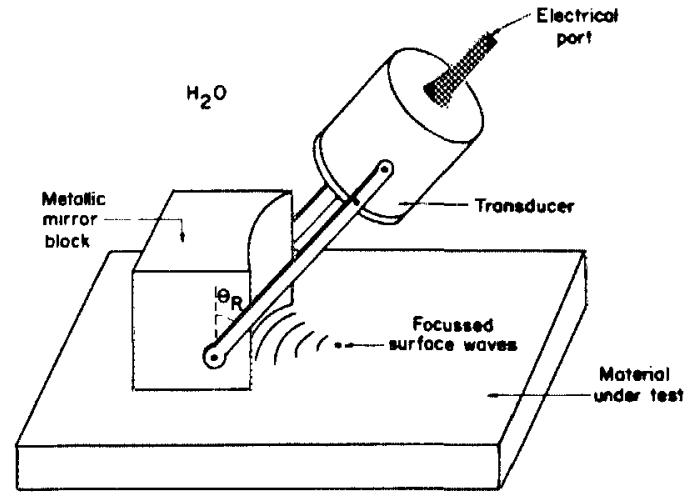

FIG. 1. Geometry to obtain a focused surface wave. 
arc radius was larger. This is because of the fact that the incidence angle selected matches the $k$ vector components along the interface. Notice that by this process all the energy in a conical wave front is converted into a single circularly converging wave front of the surface wave. This wave front will generate a diffraction limited focal point, and as a result focused surface waves are obtained with almost all the acoustic power created by the transducer.

The surface wave generated is only temporary; it will eventually leak back into the liquid medium as a bulk wave. The surface waves start leaking as soon as they are generated. If the material surface is perfectly smooth without any obstructions (i.e., no surface wave reflectors), almost none of the incident power will return to the transducer. The transducer will receive an appreciable power only if there is a surface wave reflector which changes the propagation direction of the forward traveling surface waves. Suppose that a reflector exists at focal spot. In this case the reflected surface waves will be circularly divergent. They leak into the liquid medium to reconstruct the conical wave front which originally created the surface waves. The conical wave front will get collimated in the transducer direction after the reflection from the parabolic mirror. The transducer will therefore receive acoustic power because of the presence of the reflector at the surface wave focus. If the reflector is not exactly at the surface wave focus, the reflected wave front will not be an exact replica of the incident wave front and hence the transducer output voltage will be reduced.

Optimum geometry at a particular frequency can be determined theoretically by considering Schoch displacement on the surface. A mirror involving an aperture to focal length ratio of 1.5 will require a focal length which is 0.55 of the Schoch displacement for minimum loss. Analysis predicts that the tolerance on the choice of focal length is relatively loose. An optimum configuration will result in $2.7 \mathrm{~dB}$ insertion loss. For comparison an optimum wedge transducer will have $1.78 \mathrm{~dB}$ insertion loss.

We have constructed the surface wave focusing system as described operating at $1 \mathrm{MHz}$. It consists of a 1-in.-diam PZT-4 disc transducer, a brass cylindrical mirror with a focal length of $25 \mathrm{~mm}$, and other mechanical interconnections which allows the adjustment of the position and the inclination of the transducer with respect to the mirror. The apparatus is immersed into water entirely for operation. The mirror is placed on the test piece such that the concave cylindrical surface of the mirror is perpendicular to the surface of the test piece. The system is operated in pulse echo mode and the electrical signals corresponding to the reflected waves are displayed on an oscilloscope. The apparatus is mounted on a micropositioning system, which allows the surface of the test piece to be scanned laterally. The transducer is driven by an approximately $0.2-\mu \mathrm{s}$-long voltage pulse generating an ultrasonic pulse of about $5 \mu \mathrm{s}$ long.

We used aluminum test pieces for all measurements. The Rayleigh wavelength at $1 \mathrm{MHz}$ is $2.85 \mathrm{~mm}$ in aluminum which determines the size of the diffraction limited focus and the skin thickness of the excited leaky surface waves. The thickness of the test pieces is chosen to be $10 \mathrm{~mm}$ so that the aluminum medium appears as a semi-infinite medium for all
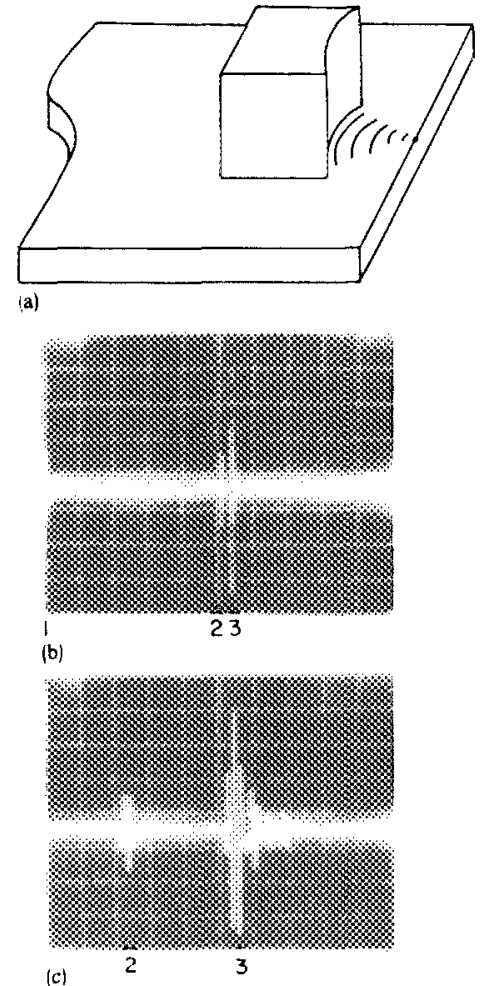

FIG. 2. Received echo pulses when the edge of the test plate is placed at the focus: (a) mirror geometry; (b) oscilloscope trace of input pulse (1), spurious pulse (2), and echo pulse from the focus (3); (c) expanded view showing just pulses (2) and (3).

practical purposes.

When the apparatus is placed such that the converging surface waves are focused on the edge of the test piece, two echoes are produced, separated by $17 \mu \mathrm{s}$ (Fig. 2). The first echo is a consequence of the fact that the generated ultrasonic wave is not a perfect plane wave, but a bounded beam. The plane wave components of the beam propagating at angles far from the Rayleigh critical angle are specularly reflected at the corner, formed by the test piece and the mirror, back to the transducer. The second pulse is significantly larger than the first and it is due to the reflection of the focused SAW at the edge. $17 \mu \mathrm{s}$ is the time required for acoustic waves to travel $50 \mathrm{~mm}$ (twice the focal length) path at the Rayleigh velocity. The adjustment of the incidence angle can be achieved by maximizing the amplitude of this pulse.

We have done various measurements to assess the performance of the focusing system. The lateral size of the focus is measured on an aluminum plate which has a vertical hole of $1 \mathrm{~mm}$ diameter. The mirror is placed a focal distance away from the hole so that the reflected Rayleigh wave amplitude is maximum. This reflected pulse amplitude is recorded as the mirror is moved laterally. It has been observed that in a 2.2-mm lateral displacement, the reflected amplitude is within $3 \mathrm{~dB}$ of the maximum. This size of the focus is in very good agreement with the expected diffraction limited size of the focus.

We have measured the insertion loss of the focusing system to be able to compare with our theoretical predictions. After correcting for the $90^{\circ} \mathrm{SAW}$ edge reflection in alumi- 
num, ${ }^{10}$ we found an insertion loss of $3.4 \mathrm{~dB}$. The expected insertion loss with our suboptimum mirror size is $3 \mathrm{~dB}$ and compares with measurement very well.

A set of measurements is performed to find the lateral spatial resolution. It was possible to resolve periodic structures with $2 \mathrm{~mm}$ periodicity. We tested the sensitivity of the system to the size of the surface flaws by approximating the flaw by a hole drilled perpendicularly to surface. The smallest ciameter hole we could drill was $0.35 \mathrm{~mm}$ in diameter, and the system was able to detect it with a signal $18.5 \mathrm{~dB}$ below an edge reflection.

The last set of measurements is made to get an understanding of the sensitivity of the focused Rayleigh waves to subsurface flaws. A special test piece is fabricated for this investigation, which contains holes with $1.5 \mathrm{~mm}$ diameter and drilled from the back face of the plate. Their depth is adjusted so that there is a different distance between the surface and the bottom of each hole. The mirror is focused on each hole and the peak amplitude of the reflected echo is recorded. It was possible to detect the subsurface hole which was $2.5 \mathrm{~mm}$ below the surface.

It can be shown that there is an inherent background signal in the system. We monitored this background signal level on a large piece of aluminum plate and compared it to the peak amplitude of the echo from an edge and observed that the dynamic range of this system is approximately $35 \mathrm{~dB}$.

Other types of conical wave generators can be used as surface wave focusing devices, provided that the angle of the cone is adjusted to equal the Rayleigh critical angle. But a $360^{\circ}$ coverage conical beam may not be very suitable, for it receives the leaked surface waves even in the absence of the surface wave reflectors. Our partial coverage axicon is more suitable, because it is essentially a zero background system, generating an output signal only when a flaw is present at the surface under investigation.

A material involving layers supports many waves other than the surface waves. These waves are known as Love waves and Stonely waves. It is possible to excite these waves selectively from the liquid side if the incidence angle of the bulk wave in the liquid is properly adjusted. The resulting wave will be focused just like the surface waves. Using this mechanism it should be possible to get much deeper penetration depth than it is possible using surface waves which are inherently associated with the surface.

Realization of the surface wave focusing axicon has shown that a very high signal level is obtained as expected. The fact that the incident power is converted almost completely into a surface wave provides this high signal level. Experimentation with the device has shown that it was quite easy to align the system. With the proposed configuration a high conversion efficiency, and therefore a high signal-tonoise ratio and sensitivity, is obtained. The resolution is limited by diffraction and hence it is determined by the wavelength of the surface waves and the maximum convergence angle. Due to their nature, the surface waves extend beneath the surface; thus the system is sensitive to variations under the surface of the material. This penetration ability is limited by the wavelength of the surface waves. A lower frequency of operation will result in a better penetration ability but lower resolution performance.

The idea presented in this letter can be extended to high frequencies with a corresponding reduction in size, to be used with acoustic microscopes as a new type of lens. The proposed system performs very close to expectations and has a promising future.

'H. Talaat and E. Burstein, J. Appl. Phys. 45, 4360 (1972).

${ }^{2}$ H. L. Bertoni and T. Tamir, IEEE Trans. Sonics Ultrason. 22, 415 (1975).

${ }^{3}$ J. Fraser, B. T. Khuri-Yakub, and G. S. Kino, Appl. Phys. Lett. 32, 698 (1978).

${ }^{4}$ H. C. Tuan, G. S. Kino, B. T. Khuri-Yakub, and A. R. Selfridge, Appl. Phys. Lett. 35, 320 (1979).

${ }^{5}$ I. R. Smith, H. K. Wickramasinghe, G. W. Farnell, and C. K. Jen, Appl. Phys. Lett. 42, 411 (1983).

${ }^{6}$ B. Nongaillard, M. Ourak, J. M. Rouvaen, M. Houze, and E. Bridoux, J. Appl. Phys. 55, 75 (1984).

${ }^{7}$ J. W. Hunt, M. Arditi, and F. S. Foster, IEEE Trans. Biomed. Eng. 30, 453 (1983).

${ }^{8}$ H. L. Bertoni and T. Tamir, Appl. Phys. 2, 157 (1973).

'Suppose that a ray is incident on a surface $A$ at an angle $\theta$. If this ray is reflected by a surface $B$ which is perpendicular to the surface $A$, the specularly reflected ray is still incident at the angle $\theta$ to the surface $A$. This can be shown by assuming an incident ray of direction $\operatorname{cosines}\left(a_{x}, \cos \theta, a_{t}\right)$ and plane $A$ as $(0,1,0)$ and plane $B$ as $(1,0,0)$ without loss of generality. Then, the reflected ray can be shown to have direction $\operatorname{cosines}\left(-a_{x}, \cos \theta, a_{z}\right)$, which has the same incidence angle to plane $A$.

${ }^{10}$ F. C. Cuozzo, E. L. Cambiaggio, J.P Damiano, and E. Rivier, IEEE Trans. Sonics Ultrason. 24, 280 (1977). 